\title{
Neuromonitoring During Surgery for Paediatric Spinal Deformity in Canada (2007)
}

\author{
Jonathan Norton, Douglas Hedden, for the Canadian Paediatric Spinal \\ Deformity Study Group
}

\begin{abstract}
Background: Neuromonitoring during paediatric (and adult) spinal deformity surgery helps to reduce the risk of both permanent and short term neurological damage. A shortage of neurophysiologists and technicians limits the availability of this service. Not all surgeons believe neuromonitoring offers neuroprotection during spinal surgery. This study aimed to document the degree to which paediatric patients undergoing spinal deformity correction surgery have their spinal cord function monitored. Methods: A questionnaire was sent electronically to all of the surgical members of the Canadian Paediatric Spinal Deformity Study Group. Results: Results were received from $9 / 9$ centres indicating that monitoring was performed in $7 / 9$ centres, with one further centre awaiting staffing. Whilst half of those centres that do monitor only monitor sensory and motor evoked potentials, the remaining centres also use EMG and EEG to assess the state of the patient intraoperatively. Conclusions: Despite a shortage of staff, most paediatric spinal deformity surgeons in Canada who wish to, are able to neurophysiologically monitor their surgical cases. Neuromonitoring appears to be becoming a standard of care, at least for paediatric spinal deformity surgery. There is an urgent need for the establishment of national standards for both technologists and interpreters, as well as training programmes for both these groups.
\end{abstract}

RÉSUMÉ: Neuromonitoring pendant la chirurgie pour déformation spinale chez les enfants au Canada (2007) Contexte : Le neuromonitoring pendant la chirurgie pour déformation spinale chez les enfants (ainsi que chez les adultes) contribue à diminuer le risque de dommages neurologiques temporaires ou permanents. Une pénurie de neurophysiologistes et de techniciens compromet la disponibilité de ce service. L'opinion des chirurgiens n'est pas unanime concernant la neuroprotection offerte par le neuromonitoring pendant la chirurgie spinale. Le but de cette étude était de documenter le niveau d'utilisation du monitoring de la fonction de la moelle épinière chez les patients d'âge pédiatrique qui subissent une chirurgie correctrice pour une déformation spinale. Méthodes : Un questionnaire a été envoyé par courrier électronique à tous les membres du Canadian Paediatric Spinal Deformity Study Group. Résultats : Neuf centres sur 9 ont répondu au questionnaire. Un monitoring était effectué dans 7 centres sur 9 et un centre a indiqué qu'on y attendait le personnel compétent pour faire ce monitoring. Bien que la moitié des centres qui font ce monitoring ne surveillent que les potentiels évoqués sensitifs et moteurs, les autres centres utilisent également l'EMG et l'EEG pour évaluer l'état du patient pendant la chirurgie. Conclusions : Malgré un manque de personnel, la plupart des chirurgiens qui opèrent des patients d'âge pédiatrique pour une déformation spinale au Canada et qui désirent un neuromonitoring pendant la chirurgie peuvent y accéder. Le neuromonitoring semble être en voie de devenir la norme en matière de soins, du moins pour la chirurgie pour déformation spinale chez les enfants. Il est impératif d'établir des standards nationaux pour les techniciens et les interprètes ainsi que des programmes de formation à leur intention.

Can. J. Neurol. Sci. 2009; 36: 47-50

The incidence of scoliosis in children under 18 years of age in Canada is $2-3 \%$ with adolescent idiopathic scoliosis being the most common cause $(\sim 80 \%)$ and occurring in neurologically normal children. Whilst the majority of these children are managed without surgery, a proportion $(\sim 10 \%)$ do require surgical correction of the deformity. Surgical correction of a long-standing spinal (skeletal) deformity is liable to place the underlying neural structures (principally the spinal cord and roots) under stress and may compromise their functional integrity. Spinal cord injury or damage clearly represents a catastrophic outcome from such surgery. In 1991 a joint position statement from the Scoliosis Research Society and the European
Spinal Deformity Society advocated the use of neuromonitoring (specifically sensory evoked potentials [SEPs]) to limit neurological damage during these procedures. ${ }^{1}$

From the Division of Pediatric Surgery, Stollery Children's Hospital, Edmonton, Alberta, Canada.

Received May 8, 2008. Final Revisions Submitted July 14, 2008. Correspondence to: Jonathan Norton, Pediatric Surgery, 2C3 North, WMC Health Sciences Centre, University of Alberta Hospital, 8440 112th Street, Edmonton, Alberta T6G 2S2, Canada. 
In many countries neuromonitoring during corrective spinal surgery is now considered a standard of care. ${ }^{2}$ Most centres now combine monitoring of both the dorsal columns (sensory evoked potentials) with monitoring of the corticospinal tract (motor evoked potentials [MEPs]). The addition of motor evoked potentials, most commonly recorded with subdermal, transcranial electrical stimulation and recorded from the muscle belly, ${ }^{2,3}$ has added a further degree of complexity to the surgical procedure, since the anaesthetic technique requires switching from halogenated agents (SEPs only) to total intravenous anaesthesia (TIVA, for MEPs). ${ }^{4-6}$ Stimulation of the motor pathways may also lead to some movement of the patient and therefore requires a close interaction between the surgical, anaesthetic and monitoring personnel. ${ }^{5}$ However, the addition of MEP monitoring allows prediction of motor deficit more accurately than SEP monitoring alone (which does not test motor pathways). ${ }^{7}$ Motor evoked potentials test the anterior portion of the spinal cord, supplied by the anterior spinal artery. Sensory evoked potentials however, test only the dorsal columns, supplied by the dorsal spinal arteries. The anterior spinal artery supplies the bulk of the spinal cord. Changes in blood flow to the spinal cord, resulting from anterior spinal artery syndrome may therefore lead to a severely disabling outcome, yet not affect the dorsal spinal columns, and hence not be detected using SEPs. ${ }^{8}$ The addition of real-time examination of EMG allows disruption to the corticospinal, and other descending pathways, to be examined. ${ }^{9-10}$ Bursts of EMG activity are seen as the alphamotoneurons are disinhibited following the loss or reduction in the descending inhibition supplied by the corticospinal tract.

Many of the studies that have examined the risk reduction associated with neuromonitoring during corrective spinal surgery (usually for scoliosis) have used adult ( $>16$ years-of-age) patients who are neurologically intact pre-operatively. In Canada, and many other countries, there is a significant shortage of clinical neurophysiologists ${ }^{11}$ and an apparent shortage of suitably trained technologists. Neuromonitoring of children undergoing corrective spinal surgery is potentially more challenging than monitoring of adults. The immature nervous system, especially the corticospinal tract, presents particular problems when monitoring. The lack of myelination of the tracts can lead to desynchronised volleys arriving at the anterior horn, and therefore not generate sufficient depolarisation of the cells therein to generate a muscle action potential. ${ }^{12,13}$ Recording of the volley within the cord directly can overcome some of these issues, ${ }^{14}$ but is not considered feasible in many cases for 'simple' scoliosis surgery. Furthermore, the child's response to anaesthesia may differ from the adult's making the anaesthetic requirements harder to accommodate. ${ }^{14}$ Many children undergoing corrective spinal deformity surgery may also be suffering from a neurological disorder (such as cerebral palsy) that may contribute to the spinal abnormality.

The Canadian Paediatric Spinal Deformity Study Group (CPSDSG) is a group of surgeons and scientists involved in the treatment of paediatric spinal deformity drawn from across Canada with representatives from each major surgical centre. At the annual meeting in 2005 the group stated that "It is the opinion of the Canadian Paediatric Spinal Deformity Study Group that the current ideal standard of care for paediatric spinal deformity correction should include SSEP and MEP monitoring." (http://cpssg.ca/). However, given the lack of suitably trained physiology technologists and neurophysiologists across the country it was not clear to what extent that was a feasible goal. As a consequence therefore, a year and a half later we distributed a survey to the members of the study group to record their current experience with neuromonitoring.

\section{Methods}

A simple questionnaire was developed that asked the respondents to document which monitoring modalities they used in both adolescent idiopathic scoliosis and neuromuscular scoliosis, and the age at which they used each modality. The questionnaire also asked about the personnel involved in the recording and interpretation of the monitoring, the equipment being used and the anaesthetic protocol. In addition we asked the respondents to provide a short reason explaining why they did, or did not monitor during spinal surgery. The questionnaire was sent to all surgeons who were members of the CPSDSG by email and non-responding centres were followed up by a personal email request or phone call. The low sample size made statistical analysis inappropriate, and this report is intended to document the state of neuromonitoring during paediatric spinal deformity surgery in Canada at a single time point (summer 2007).

\section{RESUlts}

Replies were obtained from 8/9 surgical centres. Six of the eight centres currently monitor at least some of their spinal deformity surgery. A further centre has equipment to monitor and is awaiting sufficient staffing and expects to monitor at least some cases by the end of 2007. Therefore only one center across Canada does not monitor, nor does it plan to monitor their spinal deformity surgeries.

All seven centres in which monitoring was available were able to monitor both sensory and motor evoked potentials. Monitoring when performed at a centre was done to some extent in $90-100 \%$ of cases with $50-100 \%$ of these cases at each centre having full monitoring (both SEP and MEP monitoring). Monitoring of other modalities, specifically EEG and EMG was done in only a few centres (two and three respectively). In addition only three centres (the same ones) regularly performed pedicle screw stimulation, in which electrically evoked muscle responses are used to assess the integrity of the pedicle for screw fixation.

Whilst all centres prefer the TIVA anaesthetic regime when monitoring the proportion of times in which this is given varies. Some centres only monitor when TIVA is being administered whilst in others a true TIVA, without nitrous oxide, may only be administered in $80 \%$ or less of cases. We did not ask for reasons why TIVA was not used. Whilst most centres reported that they attempted monitoring on children of any age one had a lower age limit of five years-of-age, below which they did not monitor. No centre reported a difference in monitoring protocol between neuromuscular and idiopathic scoliosis patients, despite the difficulties in recording MEPs and SEPs in children with neuromuscular diseases.

There was also considerable variation between the centres in the background of the person interpreting the evoked responses. All centres that monitored used electroneurodiagnostic technologists to perform the testing, and no centre was using 
audiologists. Interpretation of the results was done by technologists, neurosurgeons, neurologists, neurophysiologists or orthopaedic surgeons across Canada, as outlined in the Table.

\section{Table: Personnel interpreting IOM}

\begin{tabular}{ll}
\hline Providing interpretation & Number of Centres \\
Technologist & 3 \\
Neurologist & 1 \\
Neurosurgeon & 2 \\
Neurophysiologist & 3 \\
Orthopaedic Surgeon & 4 \\
Anaesthetist & 1 \\
\hline
\end{tabular}

The background of the personnel providing interpretation of the neuromonitoring, either directly in the operating room, or remotely. In most centres several people, with a variety of backgrounds, were involved in the interpretation of the results

The reasons given for monitoring varied across centres, but for those that did monitor they centered on the improved degree of patient safety/reduced risk whilst monitoring. Some surgeons also reported that they were able to achieve greater corrections during monitored cases than previously because of their increased confidence in the neurological status of the patient during the correction. The one centre that did not monitor cases cited the reason being the cost of monitoring and the absence of adverse neurological sequale in their centre. Interestingly no centre specifically commented on medico-legal issues surrounding monitoring, or not monitoring this type of surgery. In Canada, unlike the United States of America, monitoring of these cases is not regarded as a 'standard of care' with its attendant medico-legal ramifications.

\section{DISCUSSION}

Within Canada the majority of paediatric corrective spinal deformity surgery takes place with the assistance of some degree of neuromonitoring. The extent of the monitoring varies between centres, but all centres that do monitor assess both motor and sensory pathways within the spinal cord using combined sensory and motor evoked potentials.

There is currently no Canada-wide qualification for either technologists performing the testing or personnel interpreting the results. However most provinces recognize the American CINM (Certificate in Intraoperative NeuroMonitoring, provided by ABRET (American Board of Registration of Electroencephalographic and Evoked Potential Technologists)) for technologists and the D.ABNM (Diploma of the American Board of
Neurophysiological Monitoring) for physiologists who interpret the results. Appropriate qualifications/certifications for other professions (neurosurgeons/orthopaedic surgeons etc...) are not well defined. ${ }^{15}$ Most centres were not able to provide fully qualified staff at the time of the survey, and currently discussions are taking place across Canada concerning the appropriate training, certification and licensing.

Monitoring of ascending and descending sensory and motor pathways are clearly seen as important by the majority of paediatric surgeons involved in spinal deformity surgery within Canada. The other monitoring modalities are less well utilized, possibly because of their less clearly defined role. Pedicle screw stimulation can be useful in placing screws within the pedicle. Bone has higher electrical impedance than soft tissue. Since the pedicles overlie the nerve roots electrical stimulation through the pedicles will result in muscle twitches, the threshold for eliciting these twitches can be recorded. The high impedance of bone to electrical current leads to a higher threshold for eliciting a twitch when the pedicle is intact than when there is even a microscopic fracture. ${ }^{16}$ However, exact thresholds are not well defined for either thoracic pedicles or in the paediatric population. ${ }^{16}$

Monitoring of EEG provides little direct information to the surgeon, however, it can provide useful information to the anaesthetist, for whom it offers a more complex analysis than is possible with a simple 'BIS'-like system (BiSpectral index). This may be particularly useful when monitoring since the anaesthetic protocol provides the anaesthetist with less feedback on the depth of anaesthesia than a traditional, inhalational anaesthetic regime. ${ }^{6,17}$ The EEG also provides useful information when attempting to trouble-shoot some of the evoked responses. Difficulty in obtaining MEPs may be due to a burst-suppression pattern in the EEG. This pattern also reduces the number of averages needed to obtain SEPs, since there is less background noise in the EEG.

Recording of spontaneous EMG provides the neuromonitoring team and surgeon with information regarding specific root irritation and/or potential damage to the spinal cord. For instance, if a pedicle screw or hook is irritating a particular nerve root muscle discharges may be seen in a focal distribution of muscles. ${ }^{10}$ In contrast EMG discharges bilaterally, or across a wide distribution of muscles may be taken as indicator of ongoing spinal cord insult. ${ }^{9}$

Is neuromonitoring a cost-effective option? Clearly, neuromonitoring requires a considerable expense to both initiate and maintain, including equipment and staffing as well as the purchase of consumables and higher anaesthetic costs. A recent paper ${ }^{18}$ has examined in more detail the costs involved in neuromonitoring. Despite the low absolute risk of neurological damage, the high health care costs involved make monitoring cost-effective for adult patients. The health care costs for children are likely to be higher, given the increased, post-injury life expectancy. A recent survey of 180 spine surgeons across North America ${ }^{19}$ revealed that surgeons with fellowship training were more likely to monitor spine surgeries than non-fellowship trained surgeons. However while $95 \%$ of surgeons had access to SEP monitoring only $41 \%$ had access to transcranial MEPs. In the current survey, which was limited to pediatric spinal deformity 7/9 surgeons had access to both SEP and TcMEP monitoring. However fewer surgeons utilized EMG recordings 
than in the spine surgery population as a whole. This may, in part, reflect the lack of dedicated neuromonitoring technicians and physiologists working within Canada compared to the USA.

This survey looked only at the provision of monitoring during paediatric spinal deformity surgery within Canada. This survey does not examine the provision of neuromonitoring for any of the other surgical procedures for which it is indicated such as spinal or posterior fossa tumors, tethered spinal cords etc... ${ }^{5,20-22} \mathrm{We}$ anticipate that, given the membership of the CPSDSG, most of the surgeons performing the majority of the surgeries for paediatric spinal deformity within Canada were sampled by this questionnaire. Whilst in most centres surgeons were able to offer their patients neuro-monitored surgery, there remains a shortage of adequately trained personal to interpret the results, and some centres have difficulty in providing sufficient technical staff to administer the testing.

Nationally there are no standards for training of neither technologists to perform the testing nor physiologists to interpret the results. Such standards, along with a formal training programme for these critical personnel are urgently needed within Canada.

\section{ACKNOWLEDGEMENTS}

The authors thank the neuromonitoring subcommittee of the CPSDSG; Dan Borschneck, Srinivas Bulusu, Chris Cook, Jay Jarvis, Paul Moroz, Jean Ouellette, Stefan Parent, David Parsons, Chris Reilly, Vic Sahajpal and Reinhard Zeller.

\section{REFERENCES}

1. Dawson EG, Sherman JE, Kanim LEA, Nuwer MR. Spinal cord monitoring: results of the scoliosis research society and the European spinal deformity society survey. Spine. 1991; 16(8): S361-4.

2. Legatt AD. Current practice of motor evoked potential monitoring: results of a survey. J Clin Neurophysiol. 2002; 19(5):454-60.

3. MacDonald DB. Safety of intraoperative transcranial electrical stimulation motor evoked potential monitoring. J Clin Neurophysiol. 2002; 19(5):416-29.

4. Lo YL, Dan YF, Tan YE, Nurjannah S, Tan SB, Tan CT, et al. Intraoperative motor-evoked potential monitoring in scoliosis surgery: comparison of desflourane/nitrous oxide with propofol total intravenous anesthetic regimens. J Neurosurg Anesthesiol. 2006; 18(3):211-14.

5. Pajewski TN, Arlet V, Phillips LH. Current approach on spinal cord monitoring: the point of view of the neurologist, the anesthesiologist and the spine surgeon. Eur Spine J. 2007; S2:115-29.

6. DiCindio S, Schwartz DM. Anesthetic management for pediatric spinal fusion: implications of advances in spinal cord monitoring. Anesthesiol Clin North America. 2005; 23(4): 765-87.
7. MacDonald DB. Intraoperative motor evoked potential monitoring: overview and update. J Clin Monit Comput. 2006; 20(5): 347-77.

8. Nuwer MR, Daube J, Fischer C, Schramm J, Yingling CD. Neuromonitoring during surgery. Report of an IFCN Committee. Electroencephalogr Clin Neurophysiol. 1993; 87(5):263-76.

9. Skinner SA, Nagib M, Bergman TA, Maxwell RE, Msangi G. The initial use of free-running e ectromyography to detect early motor tract injury during resection of intramedullary spinal cord lesions. Neurosurgery. 2005; 56(Operative Neurosurgery 2): 299-314.

10. Holland NR. Intraoperative electromyography. J Clin Neurophys. 2002; 19(5):444-53.

11. Chan KM, Warren S, Young GB. National manpower survey on clinical neurophysiology laboratories in Canada. Can J Neurol Sci 2007; 34(2): 106.

12. Rossini PM, Barker AT, Berardelli A, Caramia MD, Caruso G, Cracco RQ, et al. Non-invasive electrical and magnetic stimulation of the brain, spinal cord and roots: basic prinicples and procedures for routine clinical application. Report of an IFCN committee. Electroencephalogr. 1994; 91:79-92.

13. Rothwell J. Techniques and mechanisms of action of transcranial stimulation of the human motor cortex. J Neurosci Methods. 1997; 74:113-22.

14. Szelenyi A, Camargo AB, Deletis V. Neurophysiological evaluation of the corticospinal tract by $\mathrm{D}$-wave recordings in young children. Childs Nerv Syst. 2003; 19(1):30-4.

15. Nuwar MR. Regulatory and medical-legal aspects of intraoperative monitoring. J Clin Neurophys. 2002; 19(5):387-95.

16. Shi YS, Binette M, Martin WH, Pearson JM, Hart RA. Electrical stimulation for intraoperative evaluation of thoracic pedicle screw placement. Spine. 2003; 28(6):595-601.

17. Miller A, Sleigh JW, Barnard J, Steyn-Ross DA. Does bispectral analysis of the electroencephalogram add anything but complexity? B J Anaesth. 2004; 92(1):8-13.

18. Sala F, Dvorak J, Faccioli F. Cost effectiveness of multimodal intraoperative monitoring during spine surgery. Eur Spine J. 2007; 16(S2):229-31.

19. Magit DP, Hilibrand AS, Kirk J, Rechtine G, Albert TJ, Vaccaro AR, et al. Questionnaire study of neuromonitoring availability and usage for spine surgery. J Spinal Disord Tech. 2007; 20(4): 282-9.

20. Sala F, Bricolo A, Faccioli F, Lanteri P, Gerosa M. Surgery for intramedullary spinal cord tumors: the role of intraoperative (neurophysiological) monitoring. Eur Spine J. 2007; S2:130-9.

21. Edmonds HL. Protective effects of neuromonitoring during cardiac surgery. Ann NY Acad Sci. 2005; 1053:12-19.

22. Dong CCJ, MacDonald DB, Akagami R, Westerberg B, AlKhani A, Kanaan I, et al. Intraoperative facial motor evoked potential monitoring with transcranial electrical stimulation during skull base surgery. Clin Neurophysiol. 2005; 116(3):588-96. 